\title{
APPLICATIONS OF THE URBAN TRAFFIC CONTROL STRATEGY TUC
}

\author{
Vaya Dinopoulou, Chistina Diakaki, Markos Papageorgiou \\ Systems and Simulation Laboratory of Technical University of Crete \\ E-mail: dinop@dssl.tuc.gr
}

\section{INTRODUCTION}

Urban traffic control systems constitute a scientific field with long-lasting and extensive research and development activities. Many methodologies have been proposed so far, but the problem of traffic-responsive network-wide signal control still lacks an adequate and efficient solution particularly under saturated traffic conditions. In fact, widely used strategies like SCOOT and SCATS, although applicable to large-scale networks, have been judged to lack a real trafficresponsive behaviour during rapidly changing conditions such as those occurring during daily business peaks or in case of incidents. This observed low performance is due to both their methodological inadequacies and their decentralised functional architecture (Diakaki, 1999). On the other hand, more advanced, recently developed, traffic-responsive strategies like OPAC, PRODYN, RHODES, etc., employ algorithms with exponential complexity, which does not permit a straightforward network-wide application. As a consequence, they use superior control levels with the aim of network-wide co-ordination, which, however, is not fully ensured due to the heuristic nature of these superior levels. Another shortcoming of the aforementioned strategies is their inability to respond adequately to saturated traffic conditions that are frequently observed in modern metropolitan areas.

In contrast to the aforementioned control strategies, TUC (Traffic-responsive $\underline{\text { Urban }} \underline{\text { Control) has }}$ been developed so as to provide co-ordinated, traffic-responsive control in large-scale urban networks, even in cases of saturated traffic conditions. This objective has been achieved via the utilisation of appropriate methodological tools that allow efficient application to large-scale networks and give rise to the following characteristics (Diakaki, 1999):

- High efficiency as suggested by the results of all the investigations under both simulated and real-life traffic conditions.

- Robustness with respect to measurement inaccuracies and disturbances.

- Reliability with respect to hardware failures (detectors, communication links, etc.).

- Generality that leads to easy applicability (via available software tools) in networks of arbitrary characteristics and dimensions.

- Extreme simplicity.

- Limited measurement requirements (one detector per significant street).

- Low computational effort. 
The aim of this paper is to briefly describe the basic philosophy, methodology and characteristics of the TUC strategy, along with application results under both simulated and real-life traffic conditions.

\section{THE TUC STRATEGY}

For TUC, three alternative control laws have been developed through the formulation of the urban traffic control problem as an optimal control problem based on a store-and-forward type of mathematical modelling (Gazis, 1963). Control objective of TUC is the minimisation and balancing of the numbers of vehicles within the streets of the controlled network. This control objective is approached through the appropriate manipulation of the green splits at urban signalised junctions, assuming given cycle times and offsets.

The first control law has been developed through the application of the Linear-Quadratic (LQ) methodology to the formulated optimal control problem. The application of this control law requires availability of nominal values of green splits. In case that such values are not available, a variation of this control law or a control law developed through the application of the LinearQuadratic-Integral (LQI) methodology to the formulated optimal control problem may be used (Diakaki, 1999).

In general, based on the store-and-forward type of modelling, the proposed approach of TUC designs (off-line) and employs (on-line) a simple control law for the traffic-responsive coordinated urban network control.

To apply TUC, availability of measurements of numbers of vehicles within streets is required. Such measurements, however, are not available unless video detection systems are installed. Otherwise, occupancy measurements collected via traditional detector loops may be used to estimate the required numbers of vehicles via the use of specially developed non-linear transformation functions.

The efficiency of the TUC strategy was tested both under simulated and real-life traffic conditions as outlined below.

\section{SIMULATION INVESTIGATIONS OF TUC}

For the simulation investigations of TUC, two existing urban networks have been utilised. Both networks have been modelled via the METACOR modelling and simulation tool, and simulation tests were conducted for several scenarios of demands and incidents with a duration of $4 \mathrm{~h}$ each. In both cases, TUC has been compared to fixed-time signal control of the considered networks.

The first application network consists of 13 signal controlled junctions and 61 links (Fig. 1) from the urban network of Glasgow, in a part of which the first field-implementation and evaluation of TUC took place within the European project TABASCO (Diakaki et al, 2000). The second 
application network consists of 17 signal controlled junctions and 79 links (Fig. 3) and represents the urban

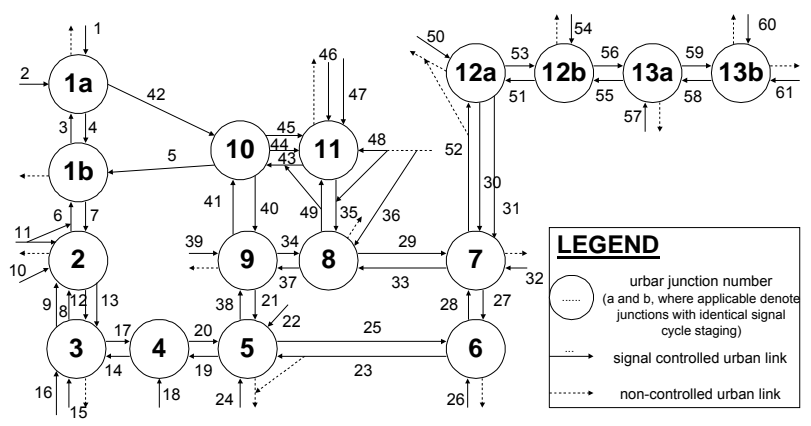

Figure 1. The first example network

network of the city centre of Chania, in two junctions of which the second field-implementation and evaluation of TUC took place within the Greek project CHANIASYN (Dinopoulou et al, 2001).

The simulation results are summarised in terms of the performance indices total waiting time at the network origins (of vehicles that wish to enter the network but their entrance is hampered due to congestion spillback at the corresponding origins; this index reflects to some extent the impact of congestion beyond the simulated network part), total travel time, total time spent (this is the sum of the two previous indices), and total fuel consumption for all vehicles during the 4-hour simulation horizons.

In both cases, the performance of TUC has been exceptional. When TUC is applied to the Glasgow network using direct measurements of numbers of vehicles within links, the total waiting time is reduced by $100 \%$, while the total travel time and the total time spent are reduced in the respective ranges $19-34 \%$ and $20-54 \%$, compared to the fixed-time signal control. The corresponding reductions in the case of the Chania network are $100 \%$ for the total waiting time, and $19-78 \%, 32-91 \%$, and $23-86 \%$ for the total travel time, the total time spent and the total fuel consumption, respectively.

Figure 2 displays an example of the time evolution of the relative occupancies $x_{z} / x_{z \text {,max }}$, where $x_{z, \max }$ is the storage capacity of network link $z$, within some selected links under fixed-time signal control and the TUC strategy applied in the Glasgow network, for one of the investigated demand scenarios. Under fixed-time signal control, congestion develops in link 34 at junction 8. This congestion spills back through links 38 at junction 9, 20 at junction 5, 17 at junction 4, and reaches link 13 at junction 3. 

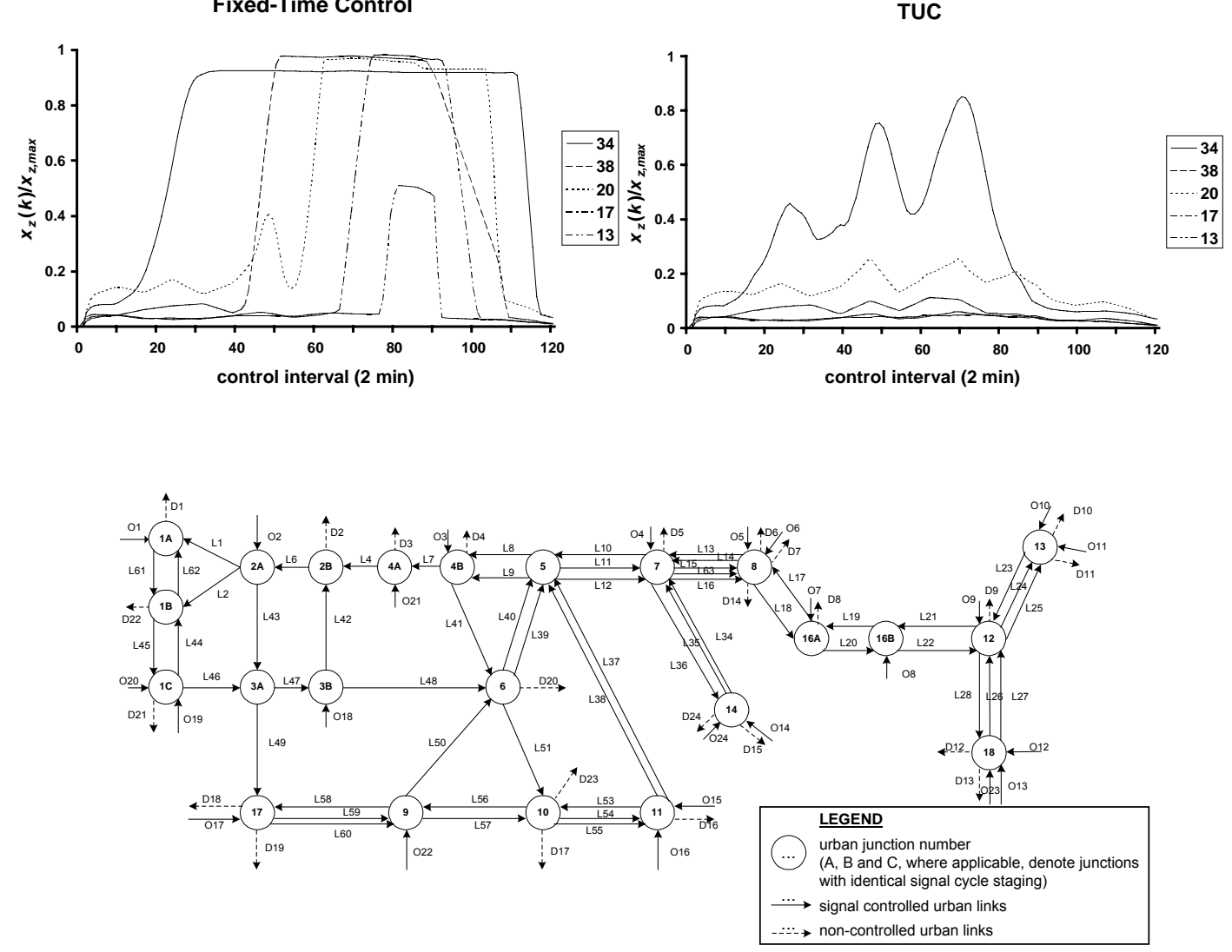

Figure 2. Comparison of fixed-time signal control and TUC for one scenario of the first example network.

Figure 2. The second example network

Under the TUC strategy the same congestion does not even reach junction 9. Similar performance is also achieved in the other investigated demand scenarios.

When TUC is applied using occupancy-based estimations of numbers of vehicles within the network streets, the achieved amelioration of the traffic conditions is lower but still significant compared to fixed-time signal control (Diakaki, 1999, Dinopoulou et all, 2000).

In conclusion, in both aforementioned investigation cases, the application of TUC largely avoids the development of extended oversaturated conditions (including gridlocks) thereby leading to a significant amelioration of the traffic conditions.

\section{FIELD IMPLEMENTATIONS AND EVALUATIONS OF TUC}

As mentioned earlier, TUC has been implemented and evaluated under real-life traffic conditions in two application networks.

The first field-implementation and evaluation was undertaken within the European project TABASCO for a part of the Glasgow urban network in Scotland (Diakaki et al, 2000). In this case, TUC was implemented as part of the integrated, traffic-responsive strategy IN-TUC (INtegrated Traffic-responsive Urban Cotnrol) (Diakaki, 1999). The main conclusion of this particular field- 
implementation and evaluation was that despite the $23 \%$-increased load due to route guidance measures, the TUC strategy managed to improve the traffic conditions of the urban network. TUC has been operational in this network since February 1998.

The second field-implementation and evaluation was undertaken within the Greek project CHANIASYN for a two-junction network of the city of Chania (Dinopoulou et al, 2001). In this case, TUC has achieved an impressive performance leading to the increase of mean speeds in the range $6-20 \%$ (13\% in average for the whole evaluation period), and the decrease of the corresponding travel times in the range $6-17 \%$ (12\% in average for the whole evaluation period). During the peak hours, the performance of TUC was much better. The mean speeds were increased in the range $7-97 \%$, while the corresponding travel times were decreased in the range 7$49 \%$, despite an observed increase of the traffic load in the range $0-12 \%$.

\section{GENERAL CONCLUSIONS AND FUTURE PLANS}

The investigations of TUC in both simulated and real-life traffic conditions indicate the high efficiency of the strategy. This high efficiency in combination with its exceptional simplicity and easy applicability suggest that TUC is probably a breakthrough in the area of urban traffic control systems.

In June 2001, started a new European project (SMART NETS) where TUC will be implemented in large parts of three European cities: Southampton (UK), Munich (Germany), and Chania (whole network). In addition, 10 further European cities (London, Paris, Athens, Prague, Copenhagen, Cork, Graz, Belfast, Glasgow, Bialystok) participate as observers in the project, with a high interest to implement TUC in the future. Within this particular project, the most recent extensions of TUC for cycle and offset control as well as provision of public transport priority will be further investigated and implemented.

Finally, preliminary simulation tests have started for the application of TUC (including all the aforementioned extensions) in parts of the urban networks of Jerusalem and Tel Aviv.

\section{REFERENCES}

Diakaki, C. (1999) Integrated Control of Traffic Flow in Corridor Networks, PhD Thesis, Technical University of Crete, Department of Production Engineering and Management, Chania.

Diakaki, C., M. Papageorgiou, T. McLean (2000) Application and evaluation of the integrated trafficresponsive urban corridor control strategy IN-TUC in Glasgow. Transportation Research Record 1727, pp. 101-111.

Dinopoulou, V., C. Diakaki, M. Papageorgiou, Z. Hourmouziadou (2000) Real-time Control Strategy of Urban Traffic: Development and Implementation in Chania (in greek). Progress Report for Phase I of the Research Project CHANIASYN of the greek Ministry of Research and Technology. 
Dinopoulou, V., C. Diakaki, G. Marinakis, M. Papageorgiou (2001) Real-time Control Strategy of Urban Traffic: Development and Implementation in Chania (in greek). Progress Report for Phase II of the Research Project CHANIASYN of the greek Ministry of Research and Technology.

Gazis, D.C., R.B. Potts (1963) The oversaturated intersection. Proceedings of the 2nd International Symposium on Traffic Theory, London, U.K., pp. 221-237. 